\title{
Upregulated IncRNA Pvt1 may be important for cardiac remodeling at the infarct border zone
}

\author{
BAIHUI LIU ${ }^{1 *}$, YUANJUAN CHENG $^{2 *}$, JIAKUN TIAN $^{1}$, LI ZHANG $^{1}$ and XIAOQIAN CUI \\ Departments of ${ }^{1}$ Emergency Medicine and ${ }^{2}$ Nursing, The Second Hospital of Jilin University, \\ Changchun, Jilin 130041, P.R. China
}

Received August 6, 2019; Accepted February 21, 2020

DOI: $10.3892 / \mathrm{mmr} .2020 .11371$

\begin{abstract}
Myocardial infarction (MI) is a leading cause of mortality due to progression to ventricular arrhythmias (VAs) or heart failure (HF). Cardiac remodeling at the infarct border zone (IBZ) is the primary contributor for VAs or HF. Therefore, genes involved in IBZ remodeling may be potential targets for the treatment of MI, but the mechanism remains unclear. The present study aimed to explain the molecular mechanisms of IBZ remodeling based on the roles of long non-coding RNAs (lncRNAs). After downloading miRNA (GSE76592) and mRNA/lncRNA (GSE52313) datasets from the Gene Expression Omnibus database, 23 differentially expressed miRNAs (DEMs), 2,563 genes (DEGs) and 168 lncRNAs (DELs) were identified between IBZ samples of MI mice and sham controls. A total of 483 DEGs were predicted to be regulated by 23 DEMs, among which Itgam, Met and TNF belonged to hub genes after five topological parameters were calculated for genes in the protein-protein interaction network. These hub genes-associated DEMs (mmu-miR-181a, mmu-miR-762) can also interact with six DELs (Gm15832, Gas5, Gm6634, Pvt1, Gm14636 and A330023F24Rik) to constitute the competing endogenous RNA (ceRNA) axes. Furthermore, a co-expression network was constructed based on the co-expression pairs between 44 DELs and 297 DEGs, in which Pvt1 and Bst1 were overlapped with the ceRNA network. Thus, Bst1-associated ceRNA (Pvt1-mmu-miR-181a-Bst1) and co-expression (Pvt-Bst1) axes were also pivotal for MI. Accordingly, Pvt1 may be a crucial lncRNA for modification of cardiac remodeling in the IBZ after MI and may function by acting as a
\end{abstract}

Correspondence to: Dr Xiaoqian Cui, Department of Emergency Medicine, The Second Hospital of Jilin University, 218 Selfstrength Street, Nanguan, Changchun, Jilin 130041, P.R. China

E-mail: cuixiaoqian18@outlook.com

*Contributed equally

Key words: myocardial infarction, infarct border zone, competing endogenous RNA, long non-coding RNAs, microRNA, co-expression
ceRNA for miR-181a to regulate $\mathrm{TNF} / \mathrm{Met} / \mathrm{Itgam} / \mathrm{Bst} 1$ or by co-expressing with Bstl.

\section{Introduction}

Myocardial infarction (MI) is one of the most frequently encountered cardiovascular diseases that can result in sudden cardiac death by inducing ventricular arrhythmias (VAs) or heart failure (HF) (1). Despite considerable advances in the treatment of $\mathrm{MI}$, the 2-year mortality rate is still high, with $49 \%$ for patients with type $2 \mathrm{MI}$ and $26 \%$ for those with type 1 MI (2). Therefore, the development of more effective therapeutic strategies for MI remains a major challenge faced by clinical cardiologists.

Accumulating evidence reveals that cardiac remodeling at the infarct border zone (IBZ) is the main contributor for the occurrence of VAs or HF $(3,4)$. Post-MI remodeling in the IBZ is characterized by compensatory cardiac hypertrophy with depressed contractile function $(3,5,6)$; extracellular matrix (ECM) rearrangement and cardiac fibrosis $(3,5)$; increased inflammatory response and cardiomyocyte apoptosis $(7,8)$ and an insufficient angiogenic response $(9,10)$. Thus, targeting the genes involved in the aforementioned processes of IBZ remodeling $(11,12)$ may be a potential approach for the treatment of MI and prevention of VAs or HF. This hypothesis has been demonstrated by previous studies. For example, Wang et al (13) directly injected pro-angiogenic vascular endothelial growth factor (VEGF) into the IBZ of MI rats and demonstrated that VEGF treatment could significantly improve cardiac function by inducing myocardial collateral vessel development and inhibiting myocardial apoptosis by decreasing the expression levels of tumor necrosis factor (TNF)- $\alpha$ and Bax. A decrease in ECM degradation enzymes (matrix metalloproteinases), but an increase in gap junction protein connexin 43 by artemisinin (14) or doxycycline (15) can improve myocardial IBZ contractility and decrease the vulnerability of VAs. Asiatic acid was reported to inhibit cardiac hypertrophy, decrease levels of inflammatory cytokines and decrease interstitial fibrosis in the IBZ of MI model rats by blocking the activation of p38 mitogen-activated protein kinase (MAPK) and ERK1/2 pathways (5). However, the molecular mechanisms of cardiac remodeling at the IBZ following MI remain unclear.

In addition to protein-coding genes, numerous studies document that long non-coding RNAs (IncRNAs; sequences 
$>200$ nucleotides in length) also play an important role in MI by acting as competing endogenous RNAs (ceRNAs) to regulate microRNA (miRNA)-mediated target repression $(16,17)$ or by directly controlling the transcription of target genes. For example, Wu et al (18) identified lncRNA ZFAS1 was significantly upregulated in the myocardium IBZ of rats at $1-48 \mathrm{~h}$ post-MI. An RNA pull-down assay indicated that ZFAS1 could directly interact with miR-150 to regulate pro-inflammatory C-reactive protein. Knockdown of ZFAS1 or overexpression of miR-150 effectively relieved MI in model rats. Hao et al (19) demonstrated that GAS5 expression was increased, while sema3a was decreased in the IBZ of the MI group. Overexpression of GAS5 could ameliorate cardiomyocyte apoptosis and decrease infarct size by downregulating the protein expression of sema3a (19). By sequencing the IBZ regions of the affected heart in porcine MI models, several novel lncRNAs expressed in an antisense orientation to myocardial transcription factors (including GATA-binding protein 4, GATA-binding protein 6 and Krüppel-like family transcription factor 6) were identified by Kaikkonen et al (20); while in the mouse MI model, Ounzain et al (21) screened hundreds of novel heart-specific IncRNAs relevant to maladaptive remodeling, such as Novlnc6, Novlnc15, Novlnc35 and Novlnc61. These lncRNAs may serve as new targets for the treatment of MI. However, to the best of our knowledge, there is a limited number of studies that focus on the roles and mechanisms of lncRNAs in the IBZ of MI.

The aim of the present study was to further screen crucial lncRNAs that are significantly differentially expressed in the IBZ of MI mice compared with sham controls by constructing lncRNA-miRNA-mRNA ceRNA and IncRNA-mRNA co-expression networks using the high throughput data deposited in public databases. The results of the present study may offer novel targets for the treatment of MI.

\section{Materials and methods}

Data collection. GSE76592 (22) and GSE52313 (21) datasets, which investigated the miRNA and lncRNA/mRNA expression level profiles in the border zone myocardium of MI model mice and sham controls, were collected from the Gene Expression Omnibus (GEO) database (http://www.ncbi.nlm. nih.gov/geo). In GSE76592 (22), the MI model was established by surgical ligation of the left anterior descending (LAD) coronary artery in mice aged 10 weeks. The ventricular septum of the areas at risk of ischemia was sampled on post-operative day 28. The sham surgeries were performed by pericardiotomy through left thoracotomy in mice aged 14 weeks to serve as the control and then the area corresponding to the border zone for MI was acquired. Total RNA was extracted from 38 MI and 11 control heart tissues and subjected to microarray analysis using GeneChip ${ }^{\circledR}$ miRNA 3.0 Arrays (platform, GPL16384; Affymetrix; Thermo Fisher Scientific, Inc.). In GSE52313 (21), the MI mouse model was also constructed by ligation of the LAD artery in 12-week old mice, and a sham operation in 12-week old mice was conducted by placing the ligature in an identical location, but not tying it. A total of four sham-operated and four infarcted heart samples were collected 2 weeks after surgery for RNA-sequencing with the
Illumina HiSeq2000 [platform, GPL9250; Illumina Genome Analyzer II (Mus musculus)].

Differential expression analysis. The normalized data were downloaded from the GEO database with the corresponding accession number. The differentially expressed miRNAs (DEMs), genes (DEGs) and lncRNAs (DELs) were identified using the Linear Models for Microarray data software package (version 3.34.0; http://www.bioconductor.org/packages/release/bioc/html/limma.html) (23) in R Bioconductor (version 3.4.1; http://www.bioconductor.org/). The Benjamini-Hochberg method for multiple testing was used to adjust $\mathrm{P}$-values to false discovery rate (FDR) (24). $\mathrm{P}<0.05$ and $\mid \log F C$ (fold-change) $>0.5$ were considered as the significant cut-off in order to screen more targets for subsequent analysis. Heatmaps were plotted using the pheatmap package (version: 1.0.8; https://cran.r-project.org/web/packages/pheatmap) based on the Euclidean distance measures.

Prediction of target encoding genes for DEMs. Prediction of target mRNAs for DEMs was performed using the miRWalk database (version 2.0; http://www.zmf.umm.uni-heidelberg. de/apps/zmf/mirwalk2) (25), which provides 12 prediction algorithms [TargetScan (version 6.2), miRanda (version 1.0), DIANA-microT (version 4.0), miRBridge (version 1.0), miRDB (version 4.0), miRMap (version 1.0), miRNAMap (version 1.0), PicTar2 (version 2.0), PITA (version 6.0), RNA22 (version 2.0), RNAhybrid (version 2.1) and miRWalk (version 1.0)]. Only target mRNAs that were predicted by at least eight different algorithms were selected. The intersections between the predicted target mRNAs and DEGs were retained, among which the DEGs that were expressed in the opposite direction to DEMs may represent the underlying downstream targets of DEMs.

Construction of a protein-protein interaction (PPI) network. In order to further screen crucial DEGs targeted by DEMs, the interactions between these DEGs were predicted using the Search Tool for the Retrieval of Interacting Genes (STRING; version 10.0; http://string db.org/) database (26). Only interactions with a combined score $>0.4$ were selected to construct the PPI network using Cytoscape (version 3.4; www.cytoscape.org/) (27). Topological features were calculated for each node (protein) in the PPI network using the CytoNCA plugin in Cytoscape software (version 3.4; http://apps.cytoscape. org/apps/cytonca) (28) to screen hub genes, including degree (DC), betweenness (BC), closeness (CC), eigenvector (EC) and sub-gragh centrality (SC).

Construction of an lncRNA ceRNA network. The DIANALncBase (version 2.0; http://carolina.imis.athena-innovation. gr/diana_tools/web/index.php?r=lncbasev2/index-predicted)(29) database was used to predict the interactions of lncRNAs with DEMs. The intersections between the predicted target lncRNAs and DELs were retained, among which the target DELs with expression in the opposite direction to DEMs may represent the underlying sponge for DEMs. The IncRNA-miRNA interaction pairs were then overlapped with the miRNA-mRNA interaction pairs according to the common miRNAs in order to generate the potential ceRNA axes, which were used to construct the ceRNA network and visualized in Cytoscape. 
Functional annotations for ceRNA network genes. Functional analysis of the genes in the ceRNA network was performed using the Database for Annotation, Visualization and Integrated Discovery (DAVID) online tool (version 6.8; http://david.abcc. ncifcrf.gov) (30). Only the enriched Gene Ontology (GO) biological process terms and Kyoto Encyclopedia of Genes and Genomes (KEGG) pathways were highlighted to represent the possible functions. $\mathrm{P}<0.05$ was considered to indicate a statistically significant difference.

Co-expression network between lncRNAs and mRNAs. In order to further screen the crucial IncRNAs and mRNAs, a co-expression network was also constructed based on the correlation between DELs and DEGs. Pearson correlation coefficients (PCC) were computed to evaluate the correlation using the WGCNA (Weighted Gene Correlation Network Analysis; https://horvath.genetics. ucla.edu/html/CoexpressionNetwork/Rpackages/WGCNA/ Tutorials/) algorithm. Only the co-expressed pairs with $|\mathrm{PCC} \geq 0.900|$ and $\mathrm{P}<0.001$ were selected to establish the co-expression network using Cytoscape. The lncRNAs and mRNAs in this co-expression network that were overlapped with those in the ceRNA network may be crucial for cardiac remodeling.

\section{Results}

Differential expression analysis. In GSE76592, 2.99\% (23/769) miRNAs were found to be differentially expressed in MI tissues compared with sham controls, including 21 upregulated and two downregulated DEMs (Table I). In GSE52313, 2,563 of 9,205 mRNAs were identified to be differentially expressed between the MI and sham control groups, including 1,705 upregulated and 858 downregulated DEGs (some are presented in Table I; all are presented in Table SI); while 91 upregulated and 77 downregulated DELs were yielded after differential analysis of 602 lncRNAs in two group samples (some are presented in Table I; all are presented in Table SII). The heatmaps indicated the MI samples could be separated from the control samples according to the expression levels of DEMs (Fig. 1A), DEGs (Fig. 1B) and DELs (Fig. 1C).

Screening of hub target mRNAs for DEMs. A total of 299 downregulated DEGs were predicted to be regulated by 21 upregulated DEMs (such as mmu-miR-337-3p-Stat5a), while 184 upregulated DEGs were predicted to be regulated by two downregulated DEMs. These 483 DEGs were mapped onto the interaction pairs collected from the STRING database. As a result, 394 DEGs (including 153 upregulated and 241 downregulated DEGs) were shown to interact with each other to generate 1,013 interactions, which were used to construct the PPI network (Fig. 2). Calculating the topological features suggested Itgam (integrin $\alpha \mathrm{M}$ ), Sod2 (superoxide dismutase 2, mitochondrial), Met (met proto-oncogene), TNF, Esr1 (estrogen receptor $1 \alpha$ ), Stat5a (signal transducer and activator of transcription 5A) and Egfr (epidermal growth factor receptor) may be hub genes as they ranked in the top 20 of all five topological parameters (Table II), indicating that their associated DEM interaction pairs may be crucial for the development of MI.
Construction of the ceRNA network. A total of 11 DEMs were predicted to interact with 32 lncRNAs, but only seven lncRNAs belonged to DELs. After further excluding the DELs with consistent expression trend to DEMs, only seven interaction pairs between two DEMs (mmu-miR-181a-5p and mmu-miR-762) and seven DELs (Gm15832, Gas5, 1810059H22Rik, Gm6634, Pvt1, Gm14636 and A330023F24Rik) remained. After overlapping the aforementioned DEMs that regulated DEGs, a total of 693 lncRNA-miRNA-mRNA interaction pairs were yielded, which included two DEMs, seven DELs and 178 DEGs (such as Met, Esr1, Sod2, TNF and Itgam). These interaction pairs were used to construct the ceRNA network (Fig. 3), in which hub gene-associated ceRNA axes, such as lncRNA Pvt1/Gm15832/A330023F24Rik/Gas5/Gm6634/Gm14636mmu-miR-181a-5p-Met/.

Esr1/TNF/Itgam and lncRNA 1810059H22Rik-mmu-m iR-762-Sod2, may be important for cardiac remodeling. In consideration of the fact that the FDR of Pvt1, Gm15832, A330023F24Rik, Gas5, Gm6634 and Gm14636 were also less than 0.05 , ceRNA axes of these six lncRNAs may be inferred to be particularly crucial for the post-MI cardiac remodeling at the IBZ.

The 178 DEGs in the ceRNA network were uploaded into the DAVID database to predict their potential functions. The results indicated 12 significant KEGG pathways, such as 'hematopoietic cell lineage (TNF and Itgam)', 'focal adhesion (Met)', 'PI3K-Akt signaling pathway (Met)', 'cytokine-cytokine receptor interaction (TNF)' and 'nicotinate and nicotinamide metabolism' [bst1 (bone marrow stromal cell antigen 1, also known as CD157)] were enriched (Table III and Fig. 4A and B). Furthermore, the hub genes enriched in the KEGG pathways were also included in 50 significant GO biological process terms, such as the 'apoptotic signaling pathway (TNF)', 'positive regulation of MAP kinase activity (TNF)', 'cell adhesion (Itgam)', 'positive regulation of the inflammatory response (TNF)', 'leukocyte cell-cell adhesion (Itgam)', 'negative regulation of cell proliferation (TNF)' and 'positive regulation of mitotic nuclear division (Met)' (Table IV and Fig. 4B).

Construction of the co-expression network. According to the threshold of $|\mathrm{PCC} \geq 0.900|$ and $\mathrm{P}<0.001,355$ co-expression pairs between 44 DELs and 297 DEGs were screened, which were used to construct the co-expression network (Fig. 5).

In order to further screen the crucial lncRNAs and mRNAs, the IncRNAs and mRNAs in co-expression and ceRNA networks were compared. The comparison results demonstrated that three lncRNAs (Pvt1, Gm15832 and A330023F24Rik) were shared between these two networks. Subsequent comparisons demonstrated that Bst1 was the common target of Pvt1, Gm15832 and A330023F24Rik, irrespective of the co-expression or ceRNA network. Thus, Bst1-associated ceRNA (Pvt1-mmu-miR-181a-5p-Bst1) and co-expression (Pvt1-Bst1) axes were inferred to be pivotal for MI.

\section{Discussion}

From the PPI and ceRNA network analyses, the present study identified six lncRNAs (Pvt1, Gm15832, A330023F24Rik, Gas5, Gm6634 and Gm14636) that sponge mmu-miR-181a-5p 


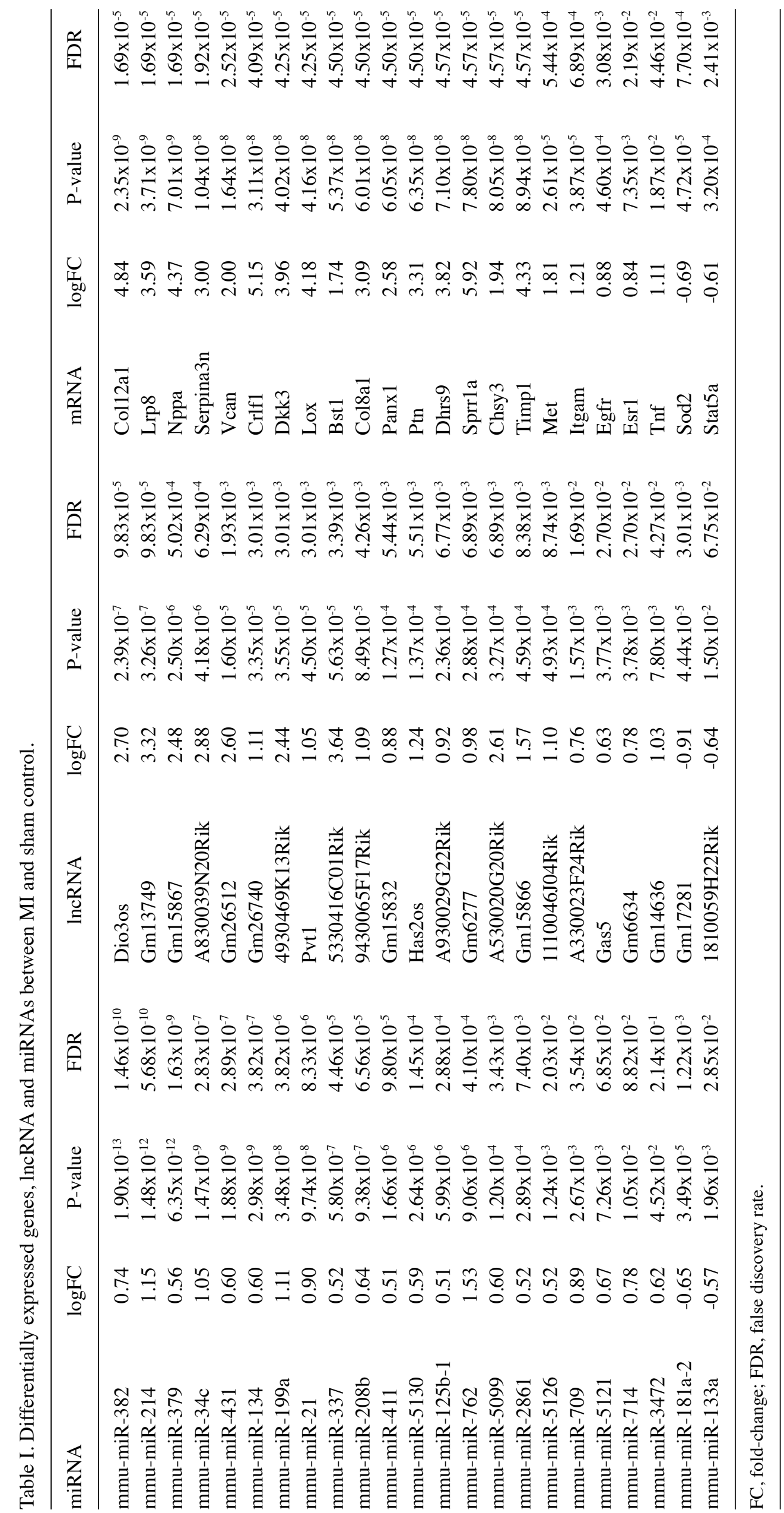



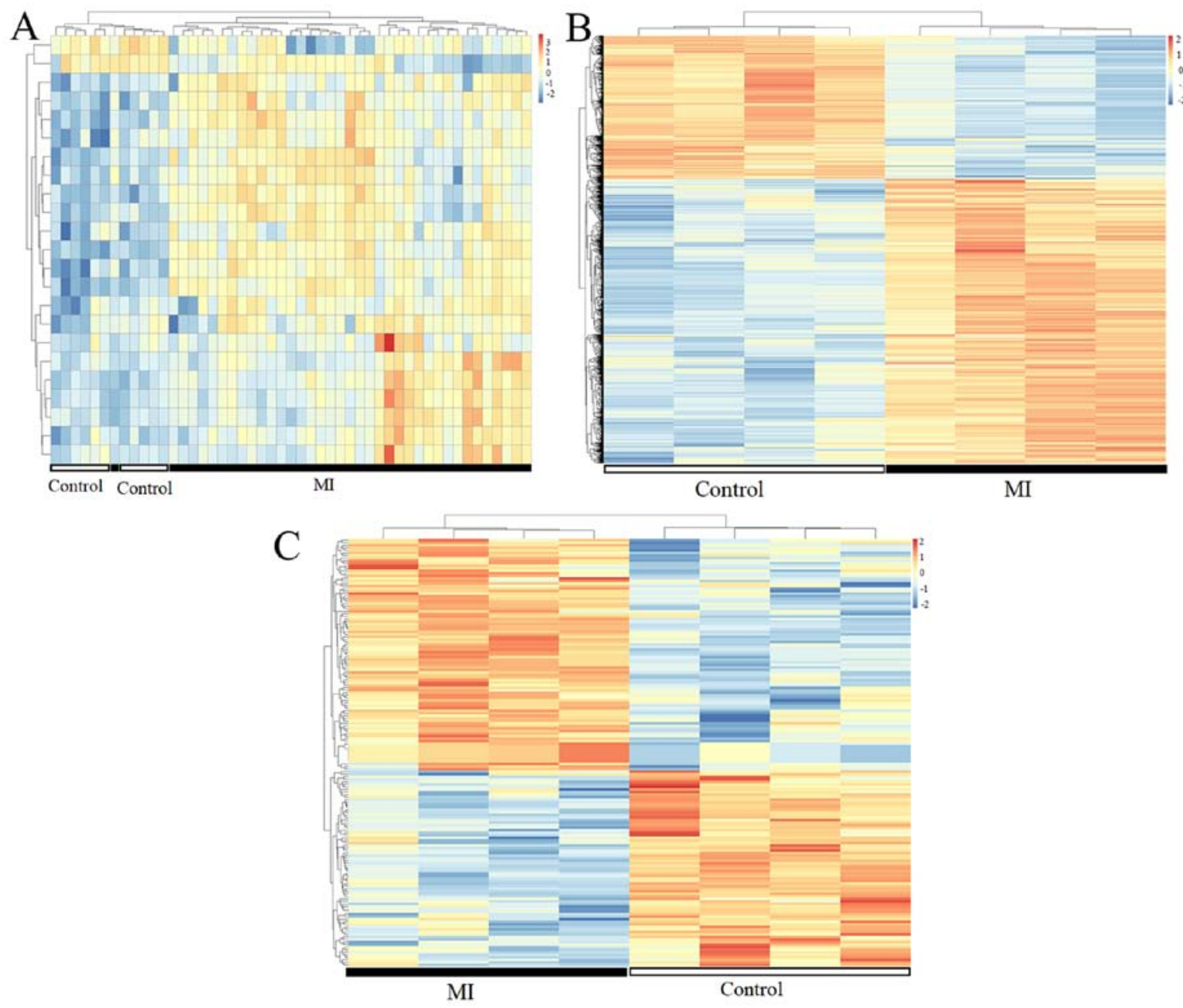

Figure 1. Heat map analysis of (A) differentially expressed microRNAs, (B) genes and (C) long non-coding RNAs. Red indicates high expression; blue indicates low expression. White box, control samples; black box, MI samples. MI, myocardial infarction.

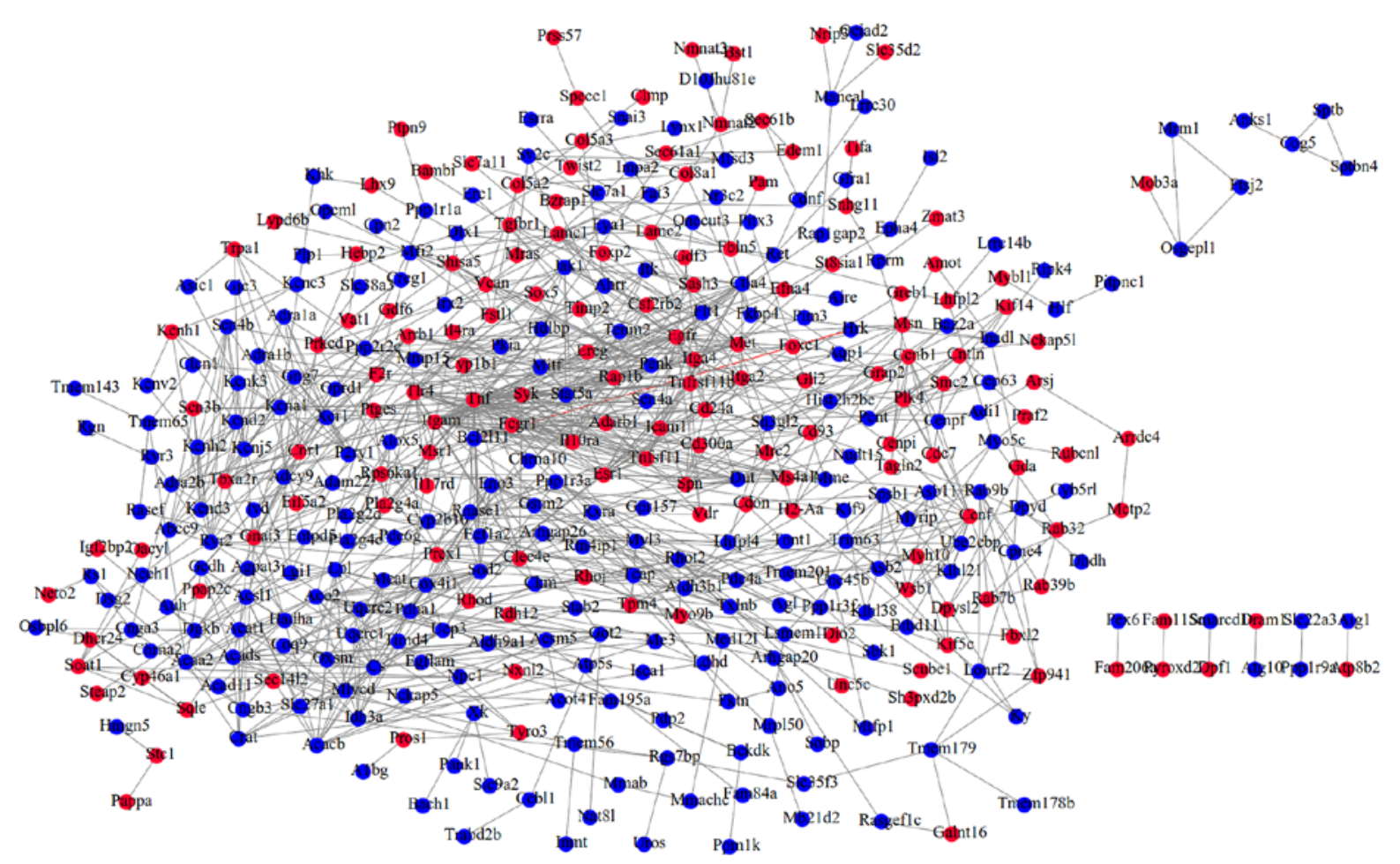

Figure 2. Protein-protein interaction network of the target genes for differentially expressed miRNAs. Red, upregulated; blue, downregulated. 
Table II. Topological features of genes in the protein-protein interaction network.

\begin{tabular}{llllllllll}
\hline ID & DC & ID & BC & ID & CC & ID & EC & ID & SC \\
\hline Tnf & 39 & Egfr & 19043.5 & Tnf & 0.0388 & Tnf & 0.356 & Tnf & 103778.8 \\
Egfr & 34 & Tnf & 14244.54 & Egfr & 0.0388 & Tlr4 & 0.288 & Itgam & 68163.56 \\
Itgam & 31 & Itgam & 12814.24 & Itgam & 0.0386 & Icam1 & 0.271 & Egfr & 60407.79 \\
Tlr4 & 23 & Ccnb1 & 9462.62 & Esr1 & 0.0385 & Cs & 0.254 & Tlr4 & 53020.38 \\
Cs & 23 & Ryr2 & 9017.83 & Sod2 & 0.0384 & Il10ra & 0.228 & Icam1 & 42583.11 \\
Esr1 & 22 & Eef1a2 & 8801.29 & Tlr4 & 0.0384 & Itgam & 0.206 & Stat5a & 34944.84 \\
Icam1 & 19 & Met & 8346.65 & Eef1a2 & 0.0384 & Ctla4 & 0.199 & Ctla4 & 32420.85 \\
Sod2 & 19 & Esr1 & 7894.01 & Dut & 0.0384 & Stat5a & 0.198 & Syk & 32246.88 \\
Stat5a & 18 & Kcna1 & 7429.03 & Icam1 & 0.0384 & Asb11 & 0.187 & Esr1 & 28649.09 \\
Syk & 18 & Sod2 & 7335.73 & Stat5a & 0.0383 & Klhl21 & 0.172 & Tnfsf11 & 24358.66 \\
Aco2 & 18 & Lamc1 & 6575.40 & Met & 0.03833 & Ube2cbp & 0.155 & Dut & 19740.2 \\
Met & 17 & Cnr1 & 6527.07 & Cnr1 & 0.0382 & Spn & 0.152 & Cd24a & 18906.82 \\
Ccnb1 & 17 & Eno3 & 5832.21 & Bcl2111 & 0.0382 & Il4ra & 0.151 & Il10ra & 18840.3 \\
Ctla4 & 16 & Lpl & 5572.44 & Cd24a & 0.0382 & Uqcrc2 & 0.149 & Itga2 & 18214.35 \\
Itga2 & 15 & Stat5a & 5350.68 & Itga2 & 0.0382 & Syk & 0.146 & Jak1 & 17525 \\
Pdha1 & 15 & Vcan & 5071.53 & Rnase1 & 0.0382 & Jak1 & 0.138 & Sod2 & 16612.51 \\
Eno3 & 15 & Aco2 & 5031.69 & Ctla4 & 0.0381 & Wsb1 & 0.136 & Fcgr1 & 15776.24 \\
Gng7 & 15 & Gnai3 & 4899.65 & Eno3 & 0.0381 & Tnfsf11 & 0.132 & Spn & 14402.02 \\
Ryr2 & 15 & Tcap & 4856.46 & Tnfsf11 & 0.0381 & Uqcrc1 & 0.131 & Met & 14210.76 \\
Hadha & 14 & Arrb1 & 4844.48 & Flt1 & 0.0381 & Cd24a & 0.122 & Bc12111 & 12157.7 \\
\hline
\end{tabular}

DC, degree centrality; BC, betweenness centrality; CC, closeness centrality; EC, eigenvector centrality; SC, subgraph centrality.
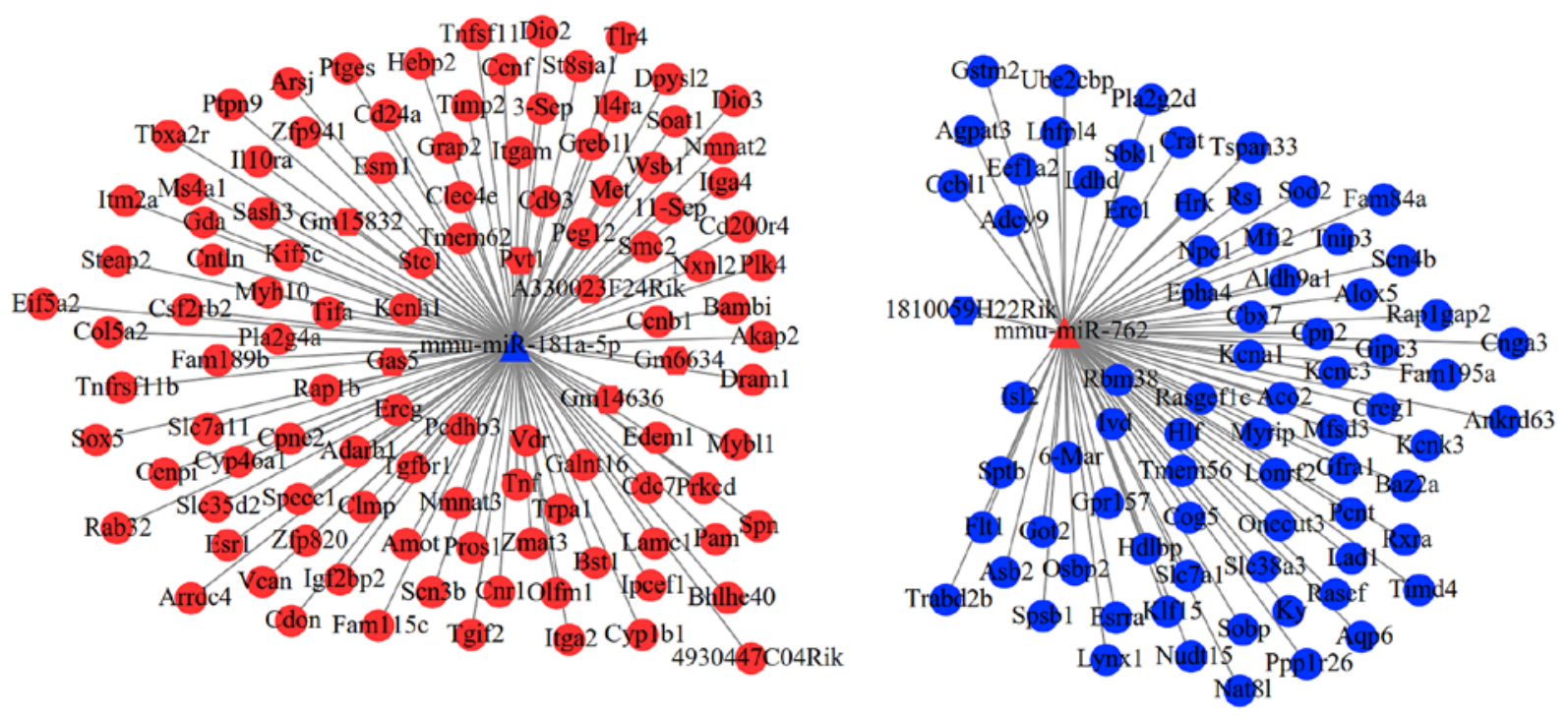

Figure 3. A competing endogenous RNA network among differentially expressed long non-coding RNAs, miRNAs and genes. Red, upregulated; blue, downregulated. Circle, differentially expressed genes; hexagon, differentially expressed long non-coding RNAs; triangle, differentially expressed miRNAs. miRNA, microRNA.

to regulate the three hub genes: TNF, Met and Itgam. These hub genes were involved in 'regulation of inflammatory response' and 'cell proliferation (TNF)', 'mitotic nuclear division (Met)' and 'cell adhesion (Itgam)'. Furthermore, based on the ceRNA and co-expression network analyses, the present study demonstrated that Pvt1 may be important for MI because it may serve a role in constituting the ceRNA axis with mmu-miR-181a-5p-Bst1 or by directly co-expressing with Bst1 to participate in nicotinate and nicotinamide metabolism.

There have been studies to prove the associations between TNF, Met and Itgam with cardiac remodeling following MI. For example, Jacobs et al (31) reported that TNF- $\alpha$ was detected in the infarct, border and remote zones of rat hearts with MI. TNF- $\alpha$ stimulation may lead to cardiac fibrosis by promoting 
Table III. KEGG pathways enriched for genes in the ceRNA network.

\begin{tabular}{llll}
\hline ID & \multicolumn{1}{c}{ Term } & P-value & \multicolumn{1}{c}{ Genes } \\
\hline mmu04640 & Hematopoietic cell lineage & $2.07 \times 10^{-4}$ & TNF, IL4RA, MS4A1, ITGA2, ITGA4, CD24A, ITGAM \\
mmu04611 & Platelet activation & $1.04 \times 10^{-2}$ & PLA2G4A, ADCY9, TBXA2R, ITGA2, RAP1B, COL5A2 \\
mmu04510 & Focal adhesion & $1.82 \times 10^{-2}$ & FLT1, MET, ITGA2, RAP1B, LAMC1, ITGA4, COL5A2 \\
mmu04913 & Ovarian steroidogenesis & $1.98 \times 10^{-2}$ & PLA2G4A, CYP1B1, ADCY9, ALOX5 \\
mmu05134 & Legionellosis & $1.98 \times 10^{-2}$ & TNF, EEF1A2, TLR4, ITGAM \\
mmu05145 & Toxoplasmosis & $2.16 \times 10^{-2}$ & TNF, IL10RA, TLR4, ALOX5, LAMC1 \\
mmu04151 & PI3K-Akt signaling pathway & $2.52 \times 10^{-2}$ & FLT1, RXRA, MET, IL4RA, ITGA2, TLR4, LAMC1, \\
& & & ITGA4, COL5A2 \\
mmu05140 & Leishmaniasis & $2.69 \times 10^{-2}$ & TNF, TLR4, ITGA4, ITGAM \\
mmu05146 & Amoebiasis & $3.07 \times 10^{-2}$ & TNF, TLR4, LAMC1, COL5A2, ITGAM \\
mmu05152 & Tuberculosis & $3.27 \times 10^{-2}$ & VDR, TNF, CLEC4E, IL10RA, TLR4, ITGAM \\
mmu04060 & Cytokine-cytokine receptor interaction & $3.57 \times 10^{-2}$ & TNFRSF11B, TNFSF11, TNF, CSF2RB2, TGFBR1, \\
& & & IL10RA, IL4RA \\
mmu00760 & Nicotinate and nicotinamide metabolism & $4.38 \times 10^{-2}$ & NMNAT3, NMNAT2, BST1
\end{tabular}

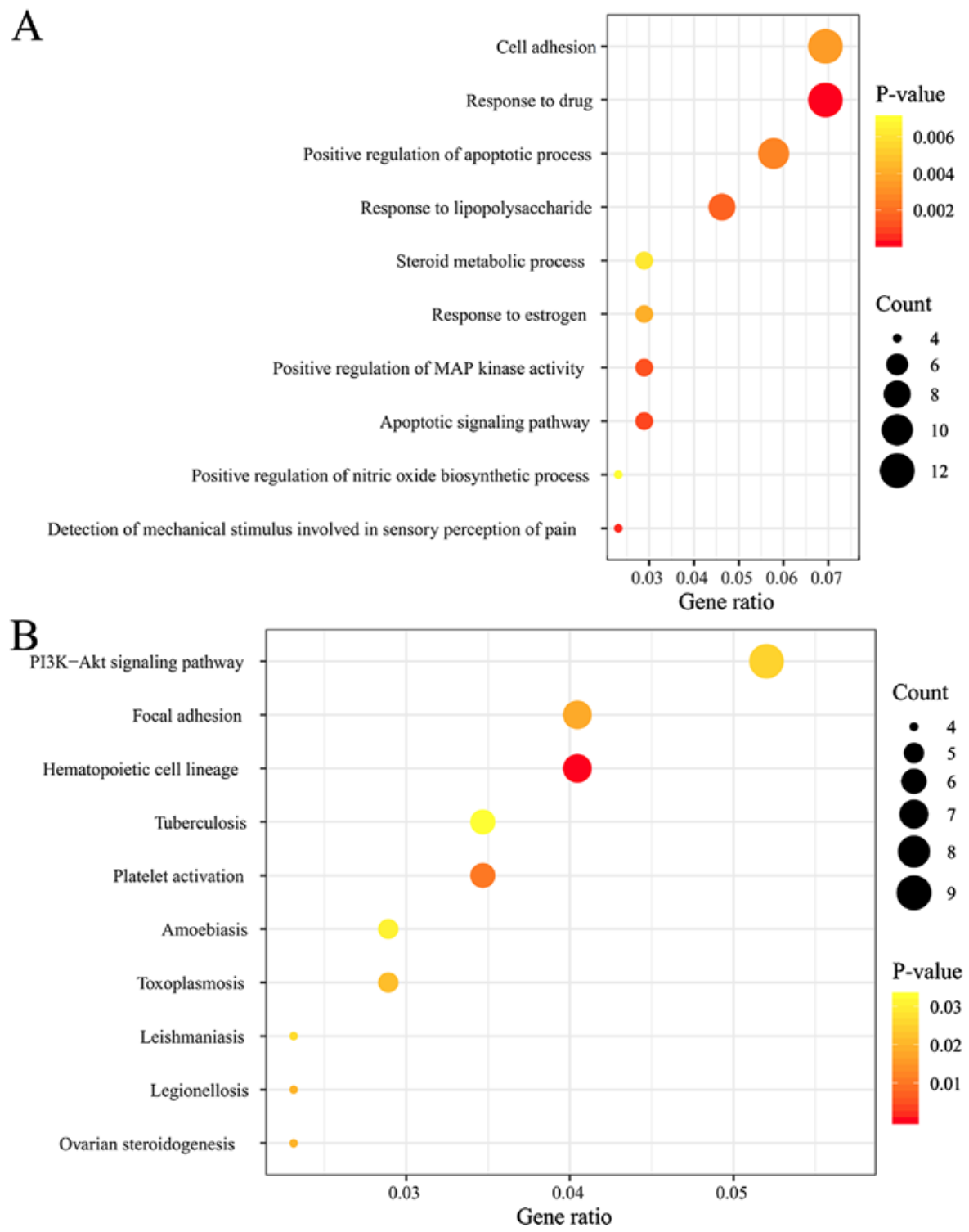

Figure 4. Function enrichment analyses for genes in the competing endogenous RNA network. (A) Gene Ontology terms; (B) Kyoto Encyclopedia of Genes and Genomes pathways. Only top 10 are listed. 
Table IV. GO terms enriched for genes in the ceRNA network.

\begin{tabular}{|c|c|c|c|}
\hline ID & Term & P-value & Genes \\
\hline GO:0042493 & Response to drug & $1.88 \times 10^{-4}$ & $\begin{array}{l}\text { CCNB1, PAM, TNFRSF11B, NPC1, TRPA1, TBXA2R, } \\
\text { ITGA2, DPYSL2, TIMP2, CBX7, KCNK3, SOD2 }\end{array}$ \\
\hline GO:0050966 & $\begin{array}{l}\text { Detection of mechanical stimulus } \\
\text { involved in sensory perception of } \\
\text { pain }\end{array}$ & $4.76 \times 10^{-4}$ & TNF, KCNA1, TRPA1, ITGA2 \\
\hline GO:0097190 & Apoptotic signaling pathway & $9.95 \times 10^{-4}$ & VDR, TNFRSF11B, TNF, CD24A, SPN \\
\hline GO:0043406 & $\begin{array}{l}\text { Positive regulation of MAP kinase } \\
\text { activity }\end{array}$ & $1.23 \times 10^{-3}$ & FLT1, TNFSF11, TNF, CD24A, PRKCD \\
\hline GO:0032496 & Response to lipopolysaccharide & $1.68 \times 10^{-3}$ & $\begin{array}{l}\text { TNFRSF11B, TNF, PTGES, CNR1, IL10RA, TBXA2R, } \\
\text { TLR4, SOD2 }\end{array}$ \\
\hline GO:0043065 & $\begin{array}{l}\text { Positive regulation of apoptotic } \\
\text { process }\end{array}$ & $2.66 \times 10^{-3}$ & $\begin{array}{l}\text { PLA2G4A, CYP1B1, TNF, EEF1A2, CNR1, ZMAT3, } \\
\text { RXRA, HRK, TLR4, PRKCD }\end{array}$ \\
\hline GO:0007155 & Cell adhesion & $3.45 \times 10^{-3}$ & $\begin{array}{l}\text { EPHA4, CYP1B1, CD93, CDON, ITGA2, VCAN, } \\
\text { LAMC1, ITGA4, CD24A, ITGAM, MYH10, RS1 }\end{array}$ \\
\hline GO:0043627 & Response to estrogen & $4.12 \times 10^{-3}$ & TNFRSF11B, TGFBR1, IL4RA, ESR1, CD24A \\
\hline GO:0008202 & Steroid metabolic process & $6.16 \times 10^{-3}$ & SOAT1, HDLBP, NPC1, CYP1B1, CYP46A1 \\
\hline GO:0045429 & $\begin{array}{l}\text { Positive regulation of nitric oxide } \\
\text { biosynthetic process }\end{array}$ & $6.98 \times 10^{-3}$ & TNF, ESR1, TLR4, SOD2 \\
\hline GO:0034220 & Ion transmembrane transport & $7.87 \times 10^{-3}$ & KCNH1, AQP6, CNGA3, KCNK3 \\
\hline GO:0045670 & Regulation of osteoclast differentiation & $9.35 \times 10^{-3}$ & ESRRA, TNFSF11, TNF \\
\hline GO:0043401 & $\begin{array}{l}\text { Steroid hormone mediated } \\
\text { signaling pathway }\end{array}$ & $1.10 \times 10^{-2}$ & VDR, ESRRA, RXRA, ESR1 \\
\hline GO:0007420 & Brain development & $1.15 \times 10^{-2}$ & $\begin{array}{l}\text { SLC38A3, KCNA1, MET, DPYSL2, SLC7A11, KCNK3, } \\
\text { MYH10 }\end{array}$ \\
\hline GO:0043410 & Positive regulation of MAPK cascade & $1.29 \times 10^{-2}$ & TNFRSF11B, FLT1, CDON, TIMP2, PRKCD \\
\hline GO:0042404 & Thyroid hormone catabolic process & $1.73 \times 10^{-2}$ & DIO2, DIO3 \\
\hline GO:0045994 & $\begin{array}{l}\text { Positive regulation of translational } \\
\text { initiation by iron }\end{array}$ & $1.73 \times 10^{-2}$ & TNF, RXRA \\
\hline GO:0050729 & $\begin{array}{l}\text { Positive regulation of inflammatory } \\
\text { response }\end{array}$ & $1.74 \times 10^{-2}$ & PLA2G4A, TNF, ITGA2, TLR4 \\
\hline GO:0046697 & Decidualization & $1.82 \times 10^{-2}$ & VDR, PLA2G4A, STC1 \\
\hline GO:0007159 & Leukocyte cell-cell adhesion & $1.82 \times 10^{-2}$ & ITGA4, CD24A, ITGAM \\
\hline GO:0006629 & Lipid metabolic process & $1.90 \times 10^{-2}$ & $\begin{array}{l}\text { SOAT1, PLA2G4A, HDLBP, NPC1, CYP46A1, PTGES, } \\
\text { ST8SIA1, CRAT, PLA2G2D, AGPAT3 }\end{array}$ \\
\hline GO:0008285 & $\begin{array}{l}\text { Negative regulation of cell } \\
\text { proliferation }\end{array}$ & $1.91 \times 10^{-2}$ & $\begin{array}{l}\text { VDR, ADARB1, CYP1B1, TNF, EREG, PTGES, RXRA, } \\
\text { TIMP2, SOD2 }\end{array}$ \\
\hline GO:0006810 & Transport & $2.52 \times 10^{-2}$ & $\begin{array}{l}\text { KCNH1, SLC38A3, HDLBP, KCNC3, MFSD3, SCN3B, } \\
\text { ZMAT3, KCNA1, IGF2BP2, AQP6, GOT2, SLC35D2, } \\
\text { OSBP2, TRPA1, MFI2, CRAT, CNGA3, KCNK3, } \\
\text { SLC7A11, COG5, SLC7A1, SCN4B, STEAP2, ERC1, } \\
\text { EIF5A2 }\end{array}$ \\
\hline GO:0023041 & Neuronal signal transduction & $2.58 \times 10^{-2}$ & KCNA1, OLFM1 \\
\hline GO:0060745 & $\begin{array}{l}\text { Mammary gland branching involved } \\
\text { in pregnancy }\end{array}$ & $2.58 \times 10^{-2}$ & VDR, ESR1 \\
\hline GO:0034628 & $\begin{array}{l}\text { 'De novo' NAD biosynthetic process } \\
\text { from aspartate }\end{array}$ & $2.58 \times 10^{-2}$ & NMNAT3, NMNAT2 \\
\hline GO:0016477 & Cell migration & $2.58 \times 10^{-2}$ & FLT1, GFRA1, LAMC1, ITGA4, BAMBI, CD24A \\
\hline GO:0045840 & $\begin{array}{l}\text { Positive regulation of mitotic nuclear } \\
\text { division }\end{array}$ & $2.61 \times 10^{-2}$ & TNF, EREG, MET \\
\hline GO:0007507 & Heart development & $2.66 \times 10^{-2}$ & PAM, TGFBR1, RXRA, VCAN, ITGA4, SOD2, MYH10 \\
\hline GO:0071407 & Cellular response to organic cyclic & $2.75 \times 10^{-2}$ & CCNB1, CYP1B1, TNF, RAP1B \\
\hline
\end{tabular}


Table IV. Continued.

\begin{tabular}{|c|c|c|c|}
\hline ID & Term & P-value & Genes \\
\hline GO:0019233 & Sensory perception of pain & $2.94 \times 10^{-2}$ & SCN3B, CNR1, TRPA1, ALOX5 \\
\hline GO:0045664 & Regulation of neuron differentiation & $2.95 \times 10^{-2}$ & CDON, DPYSL2, TIMP2 \\
\hline GO:0034765 & Regulation of ion transmembrane transport & $3.02 \times 10^{-2}$ & KCNH1, KCNC3, SCN3B, KCNA1, SCN4B \\
\hline GO:0006811 & Ion transport & $3.14 \times 10^{-2}$ & $\begin{array}{l}\text { KCNH1, SLC38A3, KCNC3, SCN3B, MFI2, } \\
\text { KCNA1, TRPA1, SCN4B, CNGA3, STEAP2, } \\
\text { KCNK3 }\end{array}$ \\
\hline GO:0055114 & Oxidation-reduction process & $3.39 \times 10^{-2}$ & $\begin{array}{l}\text { PAM, CYP1B1, CYP46A1, DIO2, DIO3, IVD, } \\
\text { LDHD, CREG1, ALOX5, STEAP2, ALDH9A1, } \\
\text { SOD2 }\end{array}$ \\
\hline GO:0045123 & Cellular extravasation & $3.43 \times 10^{-2}$ & TNF, ITGAM \\
\hline GO:0051365 & Cellular response to potassium ion starvation & $3.43 \times 10^{-2}$ & SLC38A3, HRK \\
\hline GO:0010718 & $\begin{array}{l}\text { Positive regulation of epithelial to } \\
\text { mesenchymal transition }\end{array}$ & $3.51 \times 10^{-2}$ & TGFBR1, BAMBI, OLFM1 \\
\hline GO:0048514 & Blood vessel morphogenesis & $3.70 \times 10^{-2}$ & FLT1, CYP1B1, AMOT \\
\hline GO:0019369 & Arachidonic acid metabolic process & $3.89 \times 10^{-2}$ & PLA2G4A, CYP1B1, ALOX5 \\
\hline GO:0009058 & Biosynthetic process & $4.09 \times 10^{-2}$ & GOT2, NMNAT3, NMNAT2 \\
\hline GO:0007411 & Axon guidance & $4.10 \times 10^{-2}$ & EPHA4, DPYSL2, LAMC1, CD24A, MYH10 \\
\hline GO:0008203 & Cholesterol metabolic process & $4.24 \times 10^{-2}$ & SOAT1, HDLBP, NPC1, CYP46A1 \\
\hline GO:0033591 & Response to L-ascorbic acid & $4.27 \times 10^{-2}$ & ITGA2, SOD2 \\
\hline GO:0071805 & Potassium ion transmembrane transport & $4.36 \times 10^{-2}$ & KCNH1, KCNC3, KCNA1, KCNK3 \\
\hline GO:0030199 & Collagen fibril organization & $4.50 \times 10^{-2}$ & CYP1B1, TGFBR1, COL5A2 \\
\hline GO:0001701 & In utero embryonic development & $4.65 \times 10^{-2}$ & $\begin{array}{l}\text { CCNB1, PCNT, TGFBR1, RXRA, SOX5, AMOT, } \\
\text { MYH10 }\end{array}$ \\
\hline GO:0009409 & Response to cold & $4.71 \times 10^{-2}$ & DIO2, TRPA1, SOD2 \\
\hline GO:0042127 & Regulation of cell proliferation & $4.81 \times 10^{-2}$ & $\begin{array}{l}\text { KCNH1, PLA2G4A, TNFRSF11B, ESRRA, TNF, } \\
\text { IL4RA }\end{array}$ \\
\hline GO:0008152 & Metabolic process & $4.92 \times 10^{-2}$ & $\begin{array}{l}\text { GSTM2, PAM, PLA2G4A, ACO2, IVD, ARSJ, } \\
\text { EDEM1, AGPAT3, ALDH9A1 }\end{array}$ \\
\hline
\end{tabular}

GO, Gene Ontology.

the proliferation of fibroblasts isolated from the infarct and non-infarct-region hearts. Heba et al (32) observed that the expression of TNF- $\alpha$ was increased at the border zone on days $1,4,11,28$ and 40 after MI and the expression of TNF- $\alpha$ was parallel with the development of HF after MI assessed by hemodynamic measurements. Artemisinin was demonstrated to exert beneficial effects on ventricular remodeling following MI by significantly decreasing the level of TNF- $\alpha$ at the IBZ (14). Immunohistochemistry analysis demonstrated that c-Met was intensely expressed in the border zone myocardium of the infarcted and non-infarcted region in patients with MI (33) and in certain hypertrophic myocardial cells (34). Wang et al (35) reported that compound Longmaining decoction may exert a protective effect on MI by decreasing the expression level of Itgam. Furthermore, other integrin members (such as $\alpha 5 \beta 1$ and $\alpha v \beta_{3}$ ) were also demonstrated to be upregulated to mediate abnormal vascular remodeling and pro-inflammatory macrophage polarization in endothelial cells at the IBZ following MI, further worsening cardiac hypoxia and inflammation (36,37); while downregulation of integrin- $\beta$ by erythropoietin enhanced angiogenesis, decreased inflammation and consequently improved myocardial functions (38). In line with these studies, the present analysis of GSE52313 dataset (21) also found that TNF, Met and Itgam were upregulated in the IBZ of MI model mice compared with sham controls. These findings indicate upstream regulators may also participate in cardiac remodeling by changing the expression levels of these genes.

miRNAs are 21-25 nucleotide non coding RNA molecules that bind to complementary sites in the 3'-untranslated regions of target mRNAs to repress or silence their translation. Theoretically, miRNAs (such as mmu-miR-181a-5p) that upregulate TNF, Met and Itgam, may be downregulated in the IBZ of MI model mice, notably analysis of the GSE76592 dataset (22) confirmed this in the present study. Also, previous studies demonstrated that overexpression of miR-181a, decreased cardiomyocyte cell size (i.e., hypertrophy) and apoptosis induced by high glucose, which is a common risk factor for MI (39); while miR-181a/b deficiency mice exhibited an increase in infarct size (40). The negative regulatory associations between miR-181a-5p and TNF $(41,42)$ as well as between miR-181a-5p and Met $(43,44)$ were also demonstrated 


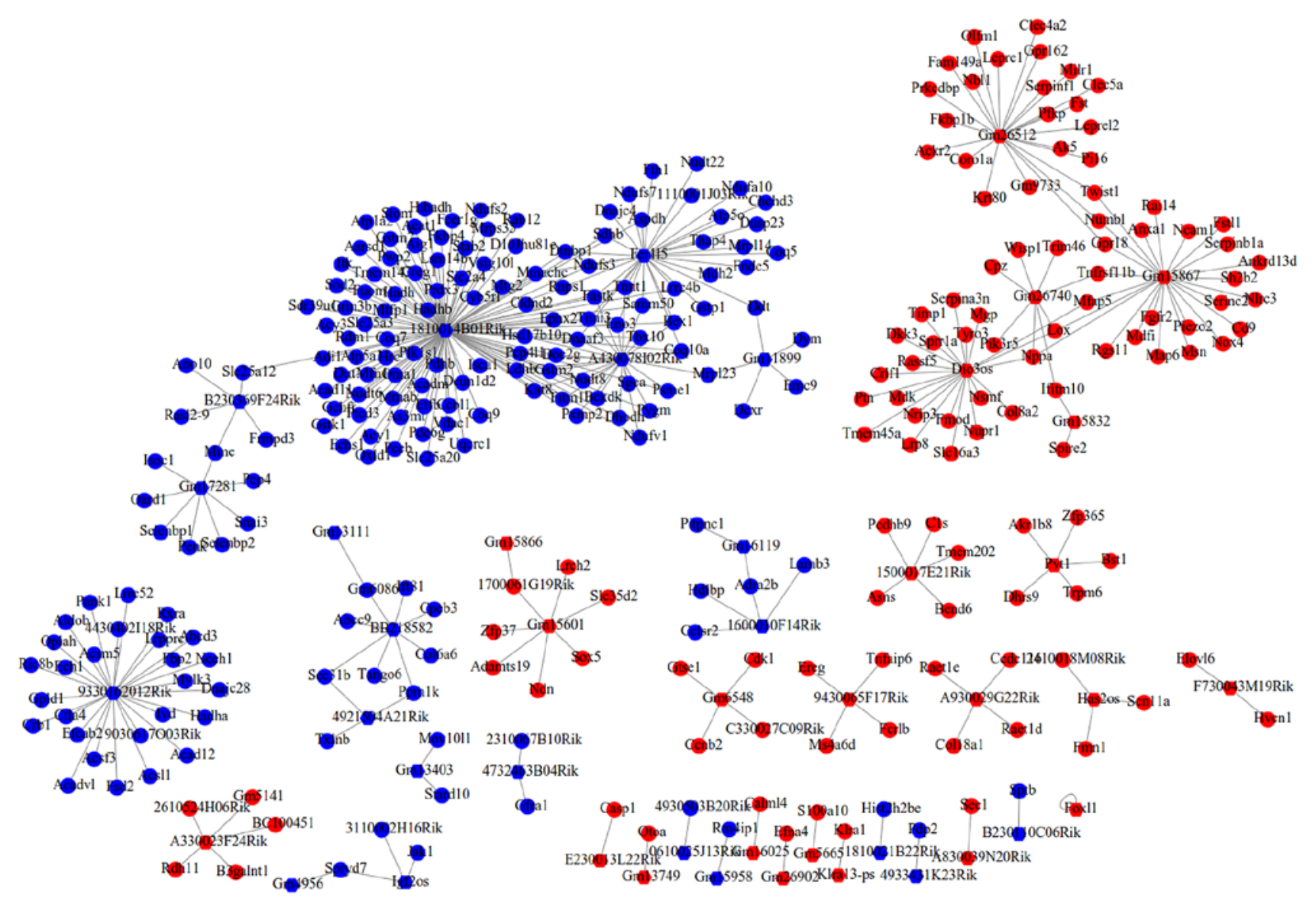

Figure 5. A co-expression network between differentially expressed long non-coding RNAs and genes. Red, upregulated; blue, downregulated. Circle, differentially expressed genes; hexagon, differentially expressed long non-coding RNAs.

to be present in immune cells and other inflammatory diseases that are important mechanisms for MI $(31,32)$. Accordingly, the miR-181a-TNF/Met interaction may be inferred to influence the development of post-MI VAs.

lncRNAs can act as ceRNAs to regulate miRNA-mediated target gene repression and thus they may also be important target genes for the treatment of MI. The present study predicted that six lncRNAs (Pvt1, Gm15832, A330023F24Rik, Gas5, Gm6634 and Gm14636) could interact with miR-181a. Among these, Gm15832 and Gm6634 may be new targets that, to the best of our knowledge, have not previously been reported in any diseases. Gm14636 was previously recorded to be upregulated to mediate the apoptotic effects of etomidate on murine leukemia RAW264.7 cells in vitro (45), which seemed to be in accordance with its potential function in MI, as Gm14636 was also observed to be upregulated in the present study. Vorinostat has previously been implicated to possess anti-inflammatory effects by increasing the expression of A330023F24Rik (46), which was not in line with the expected pro-inflammatory roles of upregulated A330023F24Rik in the IBZ in the present study. Further validation experiments should therefore be performed. Similar to the results of the present study, GAS5 had been shown to be highly expressed in the IBZ of MI model animals, but its role is controversial. Hao et al (19) suggested that overexpression of GAS5 may exert a protective effect against MI by decreasing cardiomyocyte apoptosis, while
Du et al demonstrated that silencing of GAS5 protected myocardial cells against hypoxia-induced injury (47). Taking into consideration the roles of downstream genes, the hypothesis of the present study was that high expression levels of GAS5 may be detrimental for MI as described by Du et al (47). This requires confirmation from further studies. A previous study has demonstrated that Pvt1 was upregulated in the myocardial tissues of sepsis rats, which inhibited cardiac function and promoted the secretion of inflammatory factors via activation of the MAPK/NF- $\mathrm{KB}$ pathway (48). Furthermore, Pvtl has also previously been demonstrated to be upregulated in cardiac hypertrophic model mice when compared with the sham group; while knockout of Pvt1 by siRNA significantly reduced the cardiomyocyte size (49). Consistent with these two studies, the present study also demonstrated Pvt1 was upregulated and may be involved in inflammation and cardiac hypertrophy by regulating TNF, Met and Itgam. However, to the best of our knowledge, there are a limited number of reports on the mechanisms of Pvt1 in MI and the results of the present study require additional verification.

In addition to miR-181a-5p-TNF/Met/Itgam ceRNA axes, the present study also demonstrated that Pvt1 may function in MI by acting as a ceRNA for mmu-miR-181a-5p-Bst1 or by directly co-expressing with Bst1.CD157/Bst1 encodes a protein that exhibits ADP-ribosyl cyclase (ADPRC) activity (50). ADPRCs can catalyze the conversion of nicotinamide adenine 
dinucleotide to cyclic adenosine diphosphoribose, which is a second messenger for regulating $\mathrm{Ca}(2+)$ mobilization from intracellular stores. cADPR may exert arrhythmogenic activity via its interaction with type 2 ryanodine receptors in the heart; while inhibition of cardiac ADPRC was previously reported to prevent $\mathrm{Ca}^{2+}$ overload-induced ventricular fibrillation (51). Thus, upregulation of Bst1 in the IBZ may cause VAs, which was demonstrated in the present study. Taken together, these studies on the roles of Pvt1 and miR-181a in MI along with the present study suggested these Bst-associated mechanisms may also be verifiable through further studies.

Certain limitations are present in the present study. The datasets used in the present study present limitations, such as the small sample size of the lncRNA-mRNA dataset, different platforms of miRNA and lncRNA-mRNA datasets and different sample collection time, which may bias the results. In addition, IncRNA-associated co-expression and ceRNA axes were obtained by database prediction, which may result in false positives. Therefore, wet experiments (such as quantitative PCR, luciferase assay, knockdown or overexpression) are needed to validate their interactions and roles in cardiac remodeling after MI both in vitro and in vivo.

In conclusion, the present study demonstrated that upregulated Pvt1 may be crucial for cardiac remodeling in the IBZ following MI, and may function by acting as a ceRNA for miR-181a to regulate TNF/Met/Itgam/Bst1 or by directly co-expressing with Bst1 to participate in cardiomyocyte inflammation, hypertrophy, apoptosis and contractile processes. These genes in ceRNA or co-expression axes may present targets for the treatment of MI.

\section{Acknowledgements}

Not applicable.

\section{Funding}

No funding was received.

\section{Availability of data and materials}

The datasets generated and/or analyzed during the current study are available in the GEO database in NCBI (http://www. ncbi.nlm.nih.gov/geo/).

\section{Authors' contributions}

$\mathrm{BL}, \mathrm{YC}$ and $\mathrm{XC}$ conceived and designed the original study. BL performed the bioinformatic analysis. JT and LZ contributed to the acquisition and interpretation of data. BL and YJC drafted the manuscript. XC revised the manuscript. All authors read and approved the final manuscript.

\section{Ethics approval and consent to participate}

Not applicable.

\section{Patient consent for publication}

Not applicable.

\section{Competing interests}

The authors declare that they have no competing interests.

\section{References}

1. Fahed J, Floyd KC and Tighe DA: Sudden cardiac death in postmyocardial-infarction patients. Panminerva Med 57: 71-86, 2015.

2. Saaby L, Poulsen TS, Diederichsen AC, Hosbond S, Larsen TB, Schmidt H, Gerke O, Hallas J, Thygesen K and Mickley H: Mortality rate in type 2 myocardial infarction: Observations from an unselected hospital cohort. Am J Med 127: 295-302, 2014.

3. Ma S, Ma J, Mai X, Zhao X, Guo L and Zhang M: Danqi soft capsule prevents infarct border zone remodelling and reduces susceptibility to ventricular arrhythmias in post-myocardial infarction rats. J Cell Mol Med 23: 5454-5465, 2019.

4. Chen RH, Li YG, Jiao KL, Zhang PP, Sun Y, Zhang LP, Fong XF, $\mathrm{Li} \mathrm{W}$ and Yu Y: Overexpression of Sema3a in myocardial infarction border zone decreases vulnerability of ventricular tachycardia post-myocardial infarction in rats. J Cell Mol Med 17: 608-616, 2013.

5. Huo L, Shi W, Chong L, Wang J, Zhang K and Li Y: Asiatic acid inhibits left ventricular remodeling and improves cardiac function in a rat model of myocardial infarction. Exp Ther Med 11: 57-64, 2016.

6. Wenk JF, Klepach D, Lee LC, Zhang Z, Ge L, Tseng EE, Martin A, Kozerke S, Gorman JH III, Gorman RC and Guccione JM: First evidence of depressed contractility in the border zone of a human myocardial infarction. Ann Thorac Surg 93: 1188-1193, 2012.

7. Liu X, Wan N, Zhang XJ, Zhao Y, Zhang Y, Hu G, Wan F, Zhang R, Zhu X, Xia H and Li H: Vinexin- $\beta$ exacerbates cardiac dysfunction post-myocardial infarction via mediating apoptotic and inflammatory responses. Clin Sci (Lond) 128: 923-936, 2015.

8. Takahashi T, Anzai T, Kaneko H, Mano Y, Anzai A, Nagai T, Kohno T, Maekawa Y, Yoshikawa T, Fukuda K and Ogawa S: Increased $\mathrm{C}$-reactive protein expression exacerbates left ventricular dysfunction and remodeling after myocardial infarction. Am J Physiol Heart Circ Physiol 299: H1795-H1804, 2010.

9. Song G, Li X, Shen Y, Qian L, Kong X, Chen M, Cao K and Zhang F: Transplantation of iPSc restores cardiac function by promoting angiogenesis and ameliorating cardiac remodeling in a post-infarcted swine model. Cell Biochem Biophys 71: 1463-1473, 2015

10. Zhang X, Zhu D, Wei L, Zhao Z, Qi X, Li Z and Sun D: OSM enhances angiogenesis and improves cardiac function after myocardial infarction. Biomed Res Int 2015: 317905, 2015.

11. Yang L, Gregorich ZR, Cai W, Zhang P, Young B, Gu Y, Zhang J and Ge Y: Quantitative proteomics and immunohistochemistry reveal insights into cellular and molecular processes in the infarct border zone one month after myocardial infarction. J Proteome Res 16: 2101-2112, 2017

12. Xiang F, Shi Z, Guo X, Qiu Z, Chen X, Huang F, Sha J and Chen X: Proteomic analysis of myocardial tissue from the border zone during early stage post-infarct remodelling in rats. Eur J Heart Fail 13: 254-263, 2011.

13. Wang C, Zhang B, Lin Y and Dong Y: Effects of adenovirus-mediated VEGF165 gene therapy on myocardial infarction. Ann Clin Lab Sci 48: 208-215, 2018.

14. Gu Y,Wu G, Wang X, Wang X, Wang Y and Huang C: Artemisinin prevents electric remodeling following myocardial infarction possibly by upregulating the expression of connexin 43 . Mol Med Rep 10: 1851-1856, 2014.

15. Spaulding K, Takaba K, Collins A, Faraji F, Wang G, Aguayo E, Ge L, Saloner D, Wallace AW, Baker AJ, et al: Short term doxycycline treatment induces sustained improvement in myocardial infarction border zone contractility. PLoS One 13: e0192720, 2018.

16. Sun F, Zhuang Y, Zhu H, Wu H, Li D, Zhan L, Yang W, Yuan Y, Xie Y, Yang S, et al: lncRNA PCFL promotes cardiac fibrosis via miR-378/GRB2 pathway following myocardial infarction. J Mol Cell Cardiol 133: 188-198, 2019.

17. $\mathrm{Hu} \mathrm{H}, \mathrm{Wu}$ J, Li D, Zhou J, Yu H and Ma L: Knockdown of lncRNA MALAT1 attenuates acute myocardial infarction through miR-320-Pten axis. Biomed Pharmacother 106: 738-746, 2018.

18. Wu T, Wu D, Wu Q, Zou B, Huang X, Cheng X, Wu Y, Hong K, Li P, Yang R, et al: Knockdown of long non-coding RNA-ZFAS1 protects cardiomyocytes against acute myocardial infarction via anti-apoptosis by regulating miR-150/CRP. J Cell Biochem 118: 3281-3289, 2017. 
19. Hao S, Liu X, Sui X, Pei Y, Liang Z and Zhou N: Long non-coding RNA GAS5 reduces cardiomyocyte apoptosis induced by MI through sema3a. Int J Biol Macromol 120: 371-377, 2018.

20. Kaikkonen MU, Halonen P, Liu OH, Turunen TA, Pajula J, Moreau P, Selvarajan I, Tuomainen T, Aavik E, Tavi P and Ylä-Herttuala S: Genome-wide dynamics of nascent noncoding RNA transcription in porcine heart after myocardial infarction. Circ Cardiovasc Genet 10: e001702, 2017.

21. Ounzain S, Micheletti R, Beckmann T, Schroen B, Alexanian M, Pezzuto I, Crippa S, Nemir M, Sarre A, Johnson R, et al: Genome-wide profiling of the cardiac transcriptome after myocardial infarction identifies novel heart-specific long non-coding RNAs. Eur Heart J 36: 353-368a, 2015.

22. Tsuji M, Kawasaki T, Matsuda T, Arai T, Gojo S and Takeuchi JK: Sexual dimorphisms of mRNA and miRNA in human/murine heart disease. PLoS One 12: e0177988, 2017.

23. Ritchie ME, Phipson B, Wu D, Hu Y, Law CW, Shi W and Smyth GK: limma powers differential expression analyses for RNA-sequencing and microarray studies. Nucleic Acids Res 43: e47, 2015.

24. Benjamini Y and Hochberg Y: Controlling the false discovery rate: A practical and powerful approach to multiple testing. J R Statist Soc B 57: 289-300, 1995.

25. Dweep H and Gretz N: miRWalk2.0: A comprehensive atlas of microRNA-target interactions. Nat Methods 12: 697, 2015.

26. Szklarczyk D, Franceschini A, Wyder S, Forslund K, Heller D, Huerta-Cepas J, Simonovic M, Roth A, Santos A, Tsafou KP, et al: STRING v10: Protein-protein interaction networks, integrated over the tree of life. Nucleic Acids Res 43 (Database Issue): D447-D452, 2015.

27. Kohl M, Wiese S and Warscheid B: Cytoscape: Software for visualization and analysis of biological networks. Methods Mol Biol 696: 291-303, 2011.

28. Tang Y, Li M, Wang J, Pan Y and Wu FX: CytoNCA: A cytoscape plugin for centrality analysis and evaluation of protein interaction networks. Biosystems 127: 67-72, 2015.

29. Paraskevopoulou MD, Georgakilas G, Kostoulas N, Reczko M, Maragkakis M, Dalamagas TM and Hatzigeorgiou AG: DIANA-LncBase: Experimentally verified and computationally predicted microRNA targets on long non-coding RNAs. Nucleic Acids Res 41 (Database Issue): D239-D245, 2013.

30. Huang DW, Sherman BT and Lempicki RA: Systematic and integrative analysis of large gene lists using DAVID bioinformatics resources. Nat Protoc 4: 44-57, 2009.

31. Jacobs M, Staufenberger S, Gergs U, Meuter K, Brandstätter K, Hafner M, Ertl G and Schorb W: Tumor necrosis factor-alpha at acute myocardial infarction in rats and effects on cardiac fibroblasts. J Mol Cell Cardiol 31: 1949-1959, 1999.

32. Heba G, Krzemiński T, Porc M, Grzyb J and Dembińska-Kieć A: Relation between expression of TNF alpha, iNOS, VEGF mRNA and development of heart failure after experimental myocardial infarction in rats. J Physiol Pharmacol 52: 39-52, 2001.

33. Sato T, Fujieda H, Murao S, Sato H, Takeuchi T and Ohtsuki Y: Sequential changes of hepatocyte growth factor in the serum and enhanced c-Met expression in the myocardium in acute myocardial infarction. Jpn Circ J 63: 906-908, 1999.

34. Sato T, Tani Y, Murao S, Fujieda H, Sato H, Matsumoto M, Takeuchi T and Ohtsuki Y: Focal enhancement of expression of c-Met/hepatocyte growth factor receptor in the myocardium in human myocardial infarction. Cardiovasc Pathol 10: 235-240, 2001.

35. Wang C, Bai X, Liu S, Wang J, Su Z, Zhang W, Bu D, Yan Y and Song X: RNA-seq based transcriptome analysis of the protective effect of compound longmaining decoction on acute myocardial infarction. J Pharm Biomed Anal 158: 339-345, 2018.

36. Lee SJ, Lee CK, Kang S, Park I, Kim YH, Kim SK, Hong SP, Bae H, He Y, Kubota Y and Koh GY: Angiopoietin-2 exacerbates cardiac hypoxia and inflammation after myocardial infarction. J Clin Invest 128: 5018-5033, 2018.
37. Menichetti L, Kusmic C, Panetta D, Arosio D, Petroni D, Matteucci M, Salvadori PA, Casagrande C, L'Abbate A and Manzoni L: MicroPET/CT imaging of $\alpha v \beta 3$ integrin via a novel ${ }^{63} \mathrm{Ga}-\mathrm{NOTA}-\mathrm{RGD}$ peptidomimetic conjugate in rat myocardial infarction. Eur J Nucl Med Mol Imaging 40: 1265-1274, 2013.

38. Furlani D, Klopsch C, Gäbel R, Ugurlucan M, Pittermann E, Klee D, Wagner K, Li W, Wang W, Ong LL, et al: Intracardiac erythropoietin injection reveals antiinflammatory potential and improved cardiac functions detected by forced swim test. Transplant Proc 40: 962-966, 2008.

39. Raut SK, Singh GB, Rastogi B, Saikia UN, Mittal A, Dogra N, Singh S, Prasad R and Khullar M: miR-30c and miR-181a synergistically modulate p53-p21 pathway in diabetes induced cardiac hypertrophy. Mol Cell Biochem 417: 191-203, 2016.

40. Das S, Kohr M, Dunkerly-Eyring B, Lee DI, Bedja D, Kent OA, Leung AK, Henao-Mejia J, Flavell RA and Steenbergen C: Divergent effects of miR-181 family members on myocardial function through protective cytosolic and detrimental mitochondrial microRNA targets. J Am Heart Assoc 6: e004694, 2017.

41. Zhu J, Wang FL, Wang HB, Dong N, Zhu XM, Wu Y, Wang YT and Yao YM: TNF- $\alpha$ mRNA is negatively regulated by microRNA-181a-5p in maturation of dendritic cells induced by high mobility group box-1 protein. Sci Rep 7: 12239, 2017.

42. Corsetti PP, de Almeida LA, Gonçalves ANA, Gomes MTR, Guimarães ES, Marques JT and Oliveira SC: miR-181a-5p regulates TNF- $\alpha$ and miR-21a-5p influences gualynate-binding protein 5 and IL-10 expression in macrophages affecting host control of Brucella abortus Infection. Front Immunol 9: 1331, 2018.

43. Korhan P, Erdal E and Atabey N: miR-181a-5p is downregulated in hepatocellular carcinoma and suppresses motility, invasion and branching-morphogenesis by directly targeting c-Met. Biochem Biophys Res Commun 450: 1304-1312, 2014.

44. Dong N, Tang X and Xu B: miRNA-181a inhibits the proliferation, migration, and epithelial-mesenchymal transition of lens epithelial cells. Invest Ophthalmol Vis Sci 56: 993-1001, 2015.

45. Wu RS, Wu KC, Yang JS, Chiou SM, Yu CS, Chang SJ, Chueh FS and Chung JG: Etomidate induces cytotoxic effects and gene expression in a murine leukemia macrophage cell line (RAW264.7). Anticancer Res 31: 2203-2208, 2011.

46. Ye Y, Zhao X, Lu Y, Long B and Zhang S: Varinostat alters gene expression profiles in aortic tissues from ApoE-/-mice. Hum Gene Ther Clin Dev 29: 214-225, 2018.

47. Du J, Yang ST, Liu J, Zhang KX and Leng JY: Silence of lncRNA GAS5 protects cardiomyocytes H9c2 against hypoxic injury via sponging miR-142-5p. Mol Cells 42: 397-405, 2019.

48. Feng F, Qi Y, Dong C and Yang C: PVT1 regulates inflammation and cardiac function via the MAPK/NF- $\mathrm{BB}$ pathway in a sepsis model. Exp Ther Med 16: 4471-4478, 2018.

49. Yu YH, Hu ZY, Li MH, Li B, Wang ZM and Chen SL: Cardiac hypertrophy is positively regulated by long non-coding RNA PVT1. Int J Clin Exp Pathol 8: 2582-2589, 2015.

50. Higashida H, Liang M, Yoshihara T, Akther S, Fakhrul A, Stanislav C, Nam TS, Kim UH, Kasai S, Nishimura T, et al: An immunohistochemical, enzymatic, and behavioral study of CD157/BST-1 as a neuroregulator. BMC Neuroscience 18: 35 , 2017.

51. Kannt A, Sicka K, Kroll K, Kadereit D and Gögelein H: Selective inhibitors of cardiac ADPR cyclase as novel anti-arrhythmic compounds. Naunyn Schmiedebergs Arch Pharmacol 385: 717-727, 2012.

This work is licensed under a Creative Commons Attribution-NonCommercial-NoDerivatives 4.0 International (CC BY-NC-ND 4.0) License. 